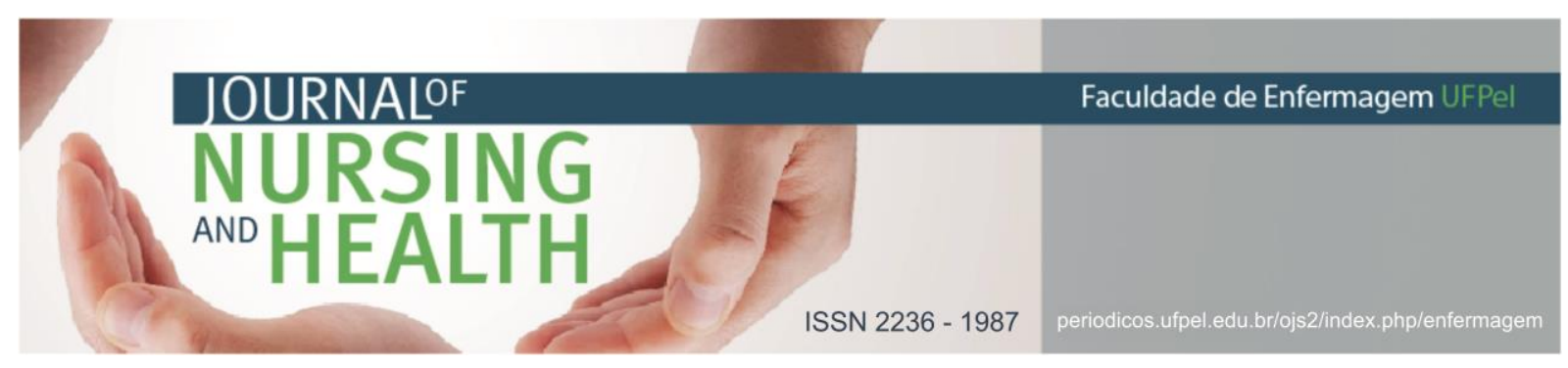

ARTIGO DE REVISÃO

\title{
A escuta de vozes na infância: uma revisão integrativa
}

\author{
Hearing voices in childhood: an integrative review \\ La escucha de voces en la infancia: una revisión integrativa \\ Cardoso, Clarissa de Souza ${ }^{1}$; Pereira, Viviane Ribeiro²; Oliveira, Naiana Alves ${ }^{3}$; Coimbra, Valéria \\ Cristina Christello ${ }^{4}$
}

Como citar este artigo: Cardoso CS, Pereira VR, Oliveira NA, Coimbra VCC. A escuta de vozes na infância: uma revisão integrativa. J. nurs. health. 2018;8(n.esp.):e188413

\section{RESUMO}

Objetivo: identificar e analisar a produção científica acerca da escuta de vozes na infância por meio da revisão integrativa. Métodos: a busca ocorreu nas bases de dados National Library of Medicine, Literatura Científica e Técnica da América Latina e Caribe e Publishier Medline, realizada no mês de março de 2018, com os descritores: criança, comportamento infantil, esquizofrenia infantil, delusões e a palavra-chave escuta de vozes, integraram a revisão 10 artigos publicados no período de 2013 a 2017. Resultados: percebe-se que as pesquisas apontaram para a predominância de saberes hegemônicos, os quais relacionam as experiências das crianças que ouvem vozes, com o diagnóstico de esquizofrenia, baseando o tratamento pela prescrição de psicofármacos. Considerações Finais: identificou-se uma produção incipiente de pesquisas na área da saúde mental que dão ênfase a temática da escuta de vozes na infância, principalmente no cenário científico brasileiro.

Descritores: Crianças; Comportamento infantil; Esquizofrenia infantil.

\section{ABSTRACT}

Objective: to identify and analyze the scientific production about the voice hearing in childhood through the integrative review. Methods: the search was carried out in the National Library of Medicine, Scientific and Technical Literature of Latin America and Caribbean and Publishier Medline, conducted in March 2018, with the descriptors: child; child behavior; schizophrenia, childhood; delusions and the keyword hearing voices, have integrated the review 10 articles published in the period from 2013 to 2017. Results: it is noticed that the researches pointed to the predominance of hegemonic knowledge, which relate the experiences of children who hear voices, with the diagnosis of schizophrenia, basing the treatment by the prescription of psychoactive drugs. Final Considerations: it was identified an incipient production of researches in the area of mental health that emphasizes the theme of hearing voices in childhood, particularly in the Brazilian scientific scenario.

Descriptors: Child; Child behavior; Schizophrenia, childhood.

1 Enfermeira. Doutoranda em Enfermagem. Universidade Federal de Pelotas (UFPEL). E-mail: cissascardoso@gmail.com http://orcid.org/0000-0002-7109-2008

2 Enfermeira. Mestre em Ciências. Universidade Federal de Pelotas (UFPEL). E-mail: viviane.ribeiropereira@gmail.com http://orcid.org/0000-0002-5553-4056

3 Enfermeira. Doutora em Ciências. Prefeitura Municipal de Pelotas/RS. E-mail: naivesoli@gmail.com http: / / orcid.org/0000-0001-5116-9082

4 Enfermeira. Doutora em Enfermagem. Universidade Federal de Pelotas (UFPEL). E-mail: valeriacoimbra@hotmail.com http://orcid.org/0000-0001-5327-0141 


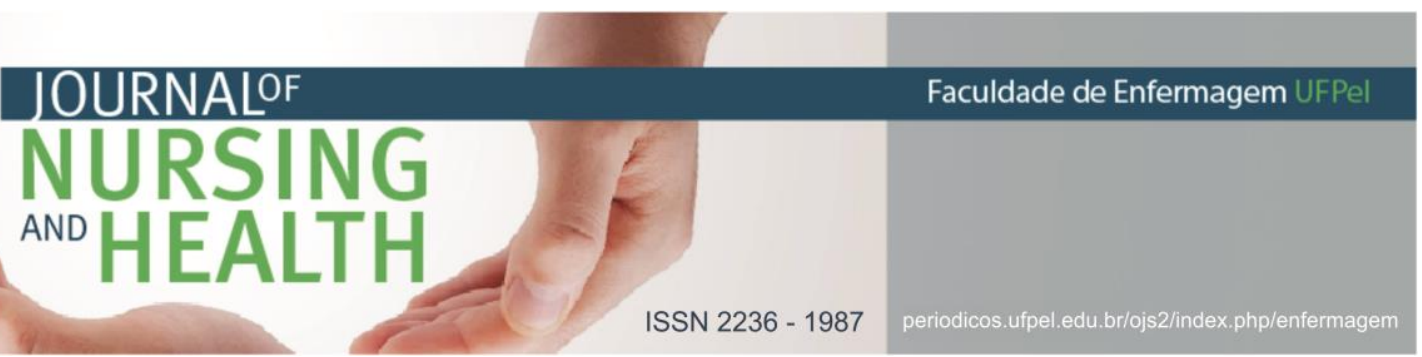

RESUMEN

Objetivo: identificar y analizar la producción científica acerca de la escucha de voces en la infancia por medio de la revisión integrativa. Métodos: la búsqueda ocurrió en las bases de datos National Library of Medicine, Literatura Científica y Técnica de América Latina y el Caribe y Publishier Medline, en el mes de marzo de 2018, con los descriptores: niño, conducta infantil, esquizofrenia infantil, delusiones y la palabra clave escucha de voces, integraron 10 artículos publicados en el período de 2013 a 2017. Resultados: predominan saberes hegemónicos, relacionados a las experiencias de los niños que oyen voces con el diagnóstico de esquizofrenia, basándose el tratamiento por la prescripción de psicofármacos. Consideraciones finales: se identificó una producción incipiente de investigaciones en el área de la salud mental que den énfasis a la temática de la escucha de voces en la infancia, principalmente en el escenario científico brasileño.

Descriptores: Niños; Comportamiento infantil; Esquizofrenia infantil.

\section{INTRODUÇÃO}

A escuta das vozes ${ }^{1}$ ou tradicionalmente chamada de alucinações auditivas são tipicamente estudadas no contexto da esquizofrenia, entrando no cenário de códigos de doenças psiquiátricas. ${ }^{2}$ Ainda, a presença de outros sintomas ou o uso de psicofármacos, direciona a investigação dos mecanismos neurocognitivos das alucinações, para a patologização. ${ }^{1}$

$\mathrm{Na}$ infância, as experiências com as alucinações, mostram uma prevalência alta em crianças, estando associadas, também, a uma série de condições médicas, isto é, acometimentos que ocorrem interferência ou lesões nas vias sensoriais periféricas que podem produzir alucinações. ${ }^{2}$ A escuta de vozes, também, pode adquirir contornos patológicos devido à família e os profissionais não conseguirem lidar com este fenômeno. ${ }^{3}$

Entretanto, existe uma parte da população geral que não necessita acessar serviços em saúde mental e lida com essas experiências sem a necessidade de cuidados específicos. ${ }^{3}$ Um dos desafios para os pesquisadores é o reconhecimento desse fenômeno como uma experiência singular vivenciada pelas crianças, e mais desafiador é compreendê-lo para além de sintomas clínicos.

Uma pesquisa realizada na Holanda 4 , com ouvidores de vozes, demonstrou que a escuta de vozes é uma experiência normal, e com o passar do tempo, em torno de $60 \%$ das crianças deixam de ouvir as vozes. ${ }^{1,4}$ Desse modo, entende-se que as crianças lidam de forma diferente com a escuta de vozes uma vez que aprendem a conviver com sua presença naturalmente, além das emoções e sentimentos envolvidos nas situações.

Tem-se que, neste sentido quando as crianças revelam suas experiências com as vozes, há uma tendência da sociedade, pais e profissionais entenderem o processo como sintomas que fazem parte de uma doença psiquiátrica, indicando que a criança não terá condições de conviver com suas experiências de escutar vozes.

Sendo assim, entendendo a necessidade de resgatar os estudos realizados acerca da escuta de vozes 


\section{JOURNALOF

por crianças que, além de sua relevância, pode subsidiar ações para o cuidado em saúde mental, esta pesquisa objetivou identificar e analisar a produção científica acerca da escuta de vozes na infância por meio da revisão integrativa. Neste sentido, elaborou-se a seguinte questão norteadora: De que forma as pesquisas tratam a temática da escuta de vozes na infância?

\section{MATERIAIS E MÉTODOS}

Trata-se de uma revisão integrativa da literatura, que tem por finalidade resgatar as publicações científicas sobre a temática da escuta de vozes na infância. A revisão integrativa caracteriza-se por ser um método que busca organizar sistematicamente estudos atuais sobre um determinado tema de investigação, a fim de incorporar resultados de pesquisas científicas relevantes ao desenvolvimento de novos estudos na área da saúde, que venham contribuir para qualidade do cuidado prestado por profissionais em sua prática clínica. ${ }^{5}$

Para isso, a elaboração da revisão integrativa, seguiu um protocolo que organizou o processo de construção a partir da identificação de um problema, a formulação de uma questão relevante, a busca das evidências, avaliação das evidências, interpretação dos dados e, por fim, a síntese e apresentação do conhecimento. ${ }^{5}$

Foi realizada a busca em duas bases de dados online para composição do estudo: Literatura LatinoAmericana e do Caribe em Ciências da Saúde (LILACS) e Publishier a Medline
(PubMed), por meio do portal da Coordenação de Aperfeiçoamento de Pessoal de Nível Superior (CAPES), no mês de março de 2018, contemplando publicações internacionais. Utilizou-se os seguintes Descritores em Ciências da Saúde (DECS) criança, comportamento infantil, esquizofrenia infantil, delusões e a palavra-chave escuta de vozes.

Para a pesquisa na PubMed todos os termos foram traduzidos para a língua inglesa segundo o Medical Subject Headings (MeSH): child, child behavior, childhood schizophrenia, delusions e voice hearing. Em ambas as buscas se utilizou os booleanos AND e OR conforme a Figura 1.

Os critérios de inclusão abordaram publicações originais nos últimos cinco anos (entre 2013 a 2017), que contextualizaram pesquisas com crianças, nos idiomas Português, Inglês e Espanhol, considerando-se os objetivos deste estudo. Como critérios de exclusão da amostra, optou-se por não utilizar artigos duplicados, resenhas, anais de congresso, artigos de opinião, artigos de reflexão, editoriais, artigos com idosos, adultos e adolescentes ouvidores de vozes, artigos que não abordassem diretamente o tema deste estudo, e artigos publicados fora do período de análise.

A busca resultou um total de 720 publicações, sendo 583 na PubMed e 137 artigos na LILACS. Após leitura de títulos e resumos, e levando em consideração os critérios de inclusão e exclusão, 710 publicações foram excluídas por não cumprirem os requisitos da revisão e não responderem à questão de pesquisa. 


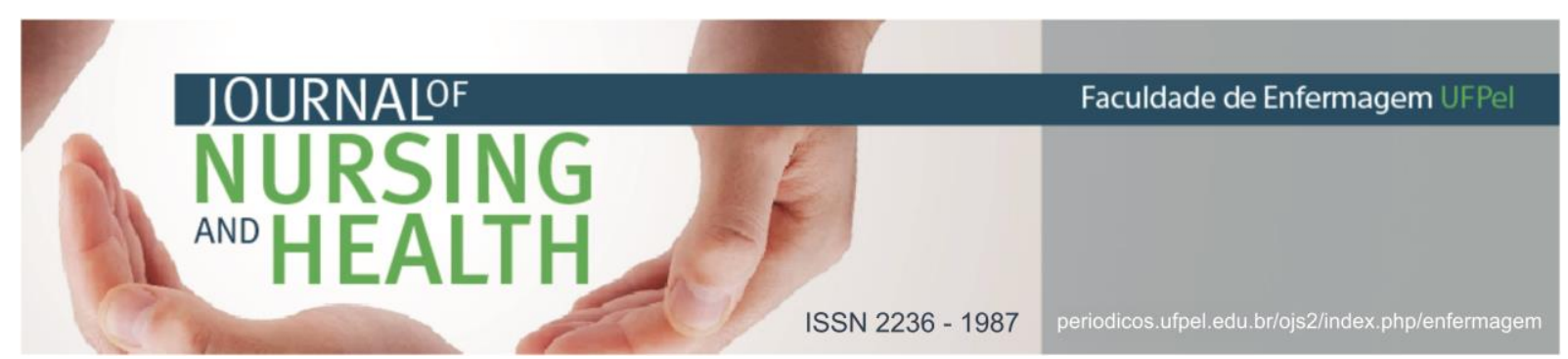

Assim, foram selecionados para a análise final 10 artigos que atenderam aos critérios e objetivos desta pesquisa.

Após ser realizada uma leitura minuciosa dos artigos na íntegra, obtidos a partir da coleta de dados, passou-se à organização dos dados, com a elaboração de um instrumento de coleta de dados contendo: nome dos autores, fonte, ano de publicação, revista e os principais resultados. Desse modo, na análise final os artigos foram identificados e verificados por dois avaliadores na busca por evidências que pudessem contribuir com a revisão.

Figura 1: Fluxograma da estratégia de busca

\section{Estratégia de busca \\ (Utilizado os mesmos descritores em português)}

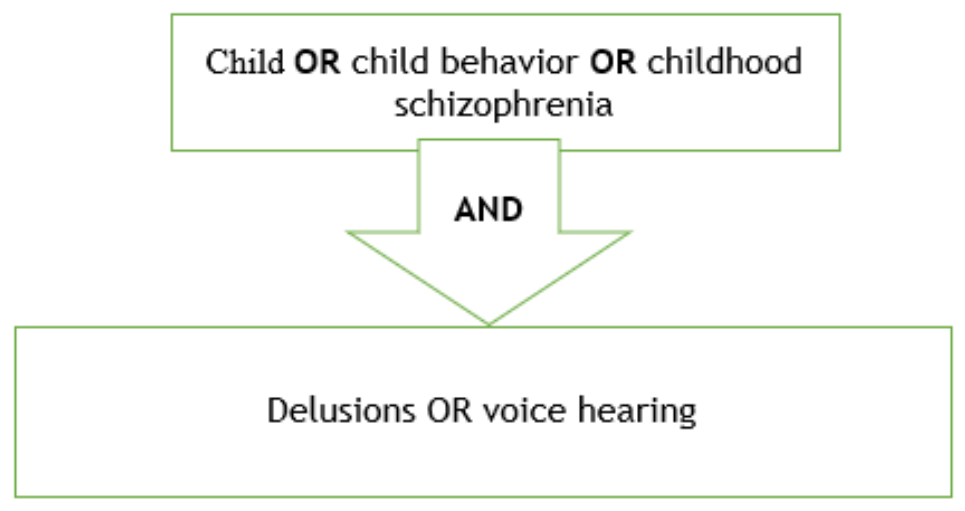

\section{RESULTADOS}

Os resultados foram organizados e apresentados em duas etapas, a primeira relacionada à caracterização dos estudos selecionados e a segunda etapa relativa à discussão dos dados encontrados.

\section{Caracterização dos estudos}

Construiu-se um quadro com as seguintes informações: nome dos autores, fonte, ano de publicação, revista e os principais resultados. Após prosseguiu-se a leitura na íntegra dos artigos para extração de informações que fornecessem subsídios para a construção da revisão integrativa proposta.

A Figura 2 apresenta os dados referentes aos artigos encontrados nas bases de dados PubMed e LILACS, entre os anos de 2013 a 2017. 
Figura 2: Artigos que compõe a amostra da Revisão integrativa, publicados nas bases de dados PubMed e LILACS, entre 2013 a 2017.

\begin{tabular}{|c|c|c|c|c|c|}
\hline $\mathrm{N}^{\circ}$ & $\begin{array}{c}\text { Nome dos } \\
\text { autores }\end{array}$ & Fonte & Ano & Revista & Principais resultados \\
\hline 1 & Tiffin, Kitchen & Pubmed & 2015 & $\begin{array}{l}\mathrm{Br} \mathrm{j} \\
\text { Psychiatri }\end{array}$ & 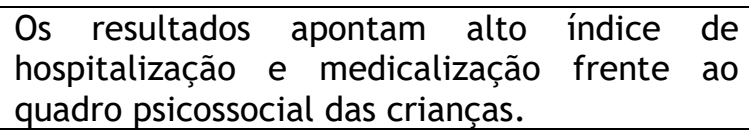 \\
\hline 2 & $\begin{array}{l}\text { Jeppesen et } \\
\text { al. }\end{array}$ & Pubmed & 2015 & $\begin{array}{l}\text { Schizophr } \\
\text { Bull }\end{array}$ & $\begin{array}{l}\text { Conclui-se que fatores genéticos (parentes de } \\
\text { primeiro grau- pai mãe e irmãos) e ambientais } \\
\text { podem contribuir para o desenvolvimento de } \\
\text { transtornos psicóticos na infância. }\end{array}$ \\
\hline 3 & Ruffel et al. & Pubmed & 2016 & $\begin{array}{l}\text { Eur Child } \\
\text { Adolesc } \\
\text { Psychiatr }\end{array}$ & $\begin{array}{l}\text { Experiências incomuns vivenciadas pelas } \\
\text { crianças e a importância do desenvolvimento } \\
\text { de abordagens e intervenções direcionadas } \\
\text { para a redução do estresse e o impacto na vida } \\
\text { cotidiana. }\end{array}$ \\
\hline 4 & Sikich & PubMed & 2013 & $\begin{array}{l}\text { Child } \\
\text { Psychiatr } \\
\text { Clin Am. }\end{array}$ & $\begin{array}{l}\text { Algumas crianças dizem que "ouvem coisas que } \\
\text { os outros não ouvem", embora raramente } \\
\text { apresentem queixas de alucinações ou } \\
\text { delírios. }\end{array}$ \\
\hline 5 & $\begin{array}{l}\text { Agnew-Blais } \\
\text { et al. }\end{array}$ & LILACS & 2015 & $\begin{array}{l}\text { Schizophr } \\
\text { Bull }\end{array}$ & $\begin{array}{l}\text { Os resultados são consistentes com o } \\
\text { comprometimento neurocognitivo ao longo da } \\
\text { primeira infância, especificamente para } \\
\text { crianças que mais tarde desenvolvem } \\
\text { esquizofrenia. }\end{array}$ \\
\hline 6 & Haug et al. & LILACS & 2015 & $\begin{array}{l}\text { Comp } \\
\text { Psychiatry }\end{array}$ & $\begin{array}{l}\text { Trauma infantil (TC) foi postulado como fator } \\
\text { de risco para o desenvolvimento de } \\
\text { esquizofrenia. }\end{array}$ \\
\hline 7 & $\begin{array}{l}\text { Ahn, Shugart, } \\
\text { Rapoport }\end{array}$ & LILACS & 2016 & $\begin{array}{l}\text { Mol } \\
\text { Psychiatr }\end{array}$ & $\begin{array}{l}\text { A esquizofrenia de início na infância (COS) é } \\
\text { uma forma rara e grave do distúrbio, mais } \\
\text { notáveis em relação a transtornos do } \\
\text { desenvolvimento pré-psicóticos e } \\
\text { anormalidades no desenvolvimento do cérebro } \\
\text { em comparação com esquizofrenia de início } \\
\text { tardio. }\end{array}$ \\
\hline 8 & $\begin{array}{l}\text { Schoroeder et } \\
\text { al. }\end{array}$ & LILACS & 2016 & $\begin{array}{l}\text { Comp } \\
\text { Psychiatr }\end{array}$ & $\begin{array}{l}\text { Experiências adversas na infância } \\
\text { exemplo, violência doméstica, perda precoce, } \\
\text { abuso sexual e físico são relatadas } \\
\text { principais fomo } \\
\text { desenvolvimento da esquizofrenia. }\end{array}$ \\
\hline 9 & $\begin{array}{l}\text { Zhou, } \\
\text { Gochman, } \\
\text { Broadnax, } \\
\text { Rapoport, Ahn }\end{array}$ & LILACS & 2016 & $\begin{array}{l}\text { Am J Med } \\
\text { Genet B }\end{array}$ & $\begin{array}{l}\text { Influência de fatores genéticos ao } \\
\text { aparecimento da esquizofrenia de início na } \\
\text { infância (COS), com alterações cromossômicas } \\
15 \text { qq13.3 herdadas paternalmente em } \\
\text { portadores de COS. }\end{array}$ \\
\hline 10 & $\begin{array}{l}\text { Lancaster } \\
\text { Hall }\end{array}$ & LILACS & 2016 & $\begin{array}{l}\mathrm{Br} \quad \mathrm{J} \\
\text { Neurology }\end{array}$ & $\begin{array}{l}\text { Alterações cerebrais influenciam } 0 \\
\text { desenvolvimento da esquizofrenia de início na } \\
\text { infância. }\end{array}$ \\
\hline
\end{tabular}




\section{IOURNALOF

Evidenciou-se, por meio desta revisão integrativa, que as publicações na grande maioria contemplam estudos com abordagens quantitativas, publicadas nas bases de dados internacionais, no idioma inglês. Para esta revisão não foram encontrados artigos publicados no idioma português e apenas dois no idioma espanhol, os quais foram descartados por não atenderem os critérios de inclusão.

Os 10 artigos selecionados estão distribuídos nos seguintes revistas internacionais: The British Journal of Psychiatry, Schizophrenia Bulletin (dois artigos), European Child Adolescent Psychiatry, Child Adolescent Psychiatric Clinics, Comprehensive Psychiatry (dois artigos), American Journal of Medical Genitics, Brain a Journal of Neurology, Molecular Psychiatry.

Foram publicados: no ano de 2013, um artigo (10\%); em 2015, quatro artigos (40\%) e 2016, cinco artigos (50\%). Com relação aos anos de 2017 e 2018 evidenciou-se a ausência de publicações que correspondesse aos objetivos do estudo.

Quanto aos conteúdos abordados nos artigos, quatro deles avaliam os fatores genéticos e ambientais e alterações cerebrais no desenvolvimento de distúrbios psicóticos na infância, ${ }^{9,11-13}$ dois artigos reconhecem a esquizofrenia na infância como uma doença rara porém grave ${ }^{13-14}$, três artigos apontam a questão do trauma e as experiências negativas e adversas vivenciadas pelas crianças como um fator de risco para doenças psicóticas $6,10,15$, um dos estudos fala da importância da intervenção direcionada para redução do estresse e diminuição do risco para doenças psíquicas em crianças, utilizando a Terapia Cognitiva Comportamental como abordagem eficaz $^{15}$, um dos artigos enfatiza a importância do diagnóstico diferenciado e precoce na identificação e tratamento da esquizofrenia ${ }^{16}$, a maioria dos artigos utiliza a entrevista semiestruturada para a análise dos dados, sendo que um deles utiliza a escala de inteligência (Teste de QI). ${ }^{17}$

Os artigos foram publicados entre os anos de 2013 (um artigo), 2015 (quatro artigos), 2016 (cinco artigos).

A discussão geral dos dados sobre a produção científica acerca da escuta de vozes na infância foi realizada de forma descritiva.

\section{DISCUSSÃO}

A maioria dos estudos ${ }^{6-10}$ apontam que as crianças que experimentam a escuta das vozes foram submetidas a situações adversas que desencadearam traumas, como o abuso físico, abuso sexual, negligência, testemunho de violência doméstica e perda de alguém próximo, como parentes ou amigos.

As pesquisas ${ }^{9,11-12}$ indicam como hipóteses para o desenvolvimento das experiências psicóticas a relação entre os diferentes fatores de risco: demográficos, genéticos e ambientais e também associam outros problemas de saúde mental como à depressão, tendência suicida, rebaixamento cognitivo e a presença de mais de uma patologia psiquiátrica. ${ }^{13}$

As experiências psicóticas podem ser um fator de risco para psicose, mas 
provavelmente apenas para um grupo da população. Para a maioria das crianças e adolescentes as vozes desaparecem com o tempo, por esta razão supõe-se que sejam um fenômeno heterogêneo com diferentes subtipos, associados ao risco de psicose, associados a outros transtornos psiquiátricos e estar dentro da faixa normal de experiências. ${ }^{7}$

Tradicionalmente estabelecem pesquisadores os previamente critérios para a avaliação das experiências, como exemplo: questionários de autoavaliação, no entanto sem utilizar instrumentos que apontem na direção de experiências tão sutis e subjetivas. Considera-se a escuta das vozes um assunto complexo, principalmente quando envolve a experiência das crianças, neste sentido o desenho do estudo e a metodologia do processo de avaliação podem ter impacto significativo nos resultados obtidos. $^{7-8}$ São poucos os trabalhos mais detalhados que avaliam especificamente como experiências auditivas. Acredita-se que o principal fator que contribui para essa situação é a compreensão incompleta desse fenômeno que é a escuta das vozes. ${ }^{7}$

Percebe-se que as pesquisas são orientadas a partir de saberes hegemônicos relacionando as experiências das crianças com 0 diagnóstico de esquizofrenia e por esta razão dando ênfase para um tratamento específico e baseado no uso de antipsicóticos. ${ }^{14-15}$

Para além de diagnosticar as alucinações auditivas e/ou visuais, diversos autores ${ }^{1,8,16}$ dialogam com a necessidade de reconhecimento precoce e tratamento eficaz como medida para a redução da incapacidade e sofrimento que podem perdurar na vida adulta. Constata-se que uma grande proporção de crianças em sofrimento mental não recebe o tratamento de que necessitam. ${ }^{8,16} \mathrm{~A}$ importância de reconhecer precocemente o que a criança enfrenta repercute na maneira como ela fará o enfrentamento das vozes.

Um dos estudos ${ }^{17}$ indicou a existência entre o desenvolvimento da esquizofrenia e a baixa cognição na primeira infância, sabe-se que o sofrimento mental causa um rebaixamento no funcionamento neurológico de crianças.

Destaca-se o estudo realizado no Reino Unido ${ }^{14}$, no qual participaram oito crianças com risco moderado para violência $(n=5)$; três com risco moderado para automutilação; sete apresentavam comorbidades psiquiátricas, entre as mais comuns: o transtorno do espectro do autismo. Porém, chama-se atenção para o número de internações psiquiátricas, pois quatro já haviam sido internadas até 0 início do estudo. Durante a realização da pesquisa, houve pelo menos uma internação hospitalar para cinco crianças concomitante a realização do estudo. Apenas um participante não recebeu medicação psiquiátrica e sete foram tratados com pelo menos dois medicamentos diferentes, o psicofármaco mais utilizado foi a clozapina. A partir destes dados pode-se perceber a necessidade de uma mudança na maneira de conduzir os casos, ${ }^{10}$ sobretudo de crianças ouvidoras de vozes, pois a maioria delas apresentou 


\section{JOURNALOF \\ NURSING \\ aNOHEALTH}

uma piora significativa no quadro psicossocial. ${ }^{17}$

Nota-se que a experiência com a escuta de vozes na infância é um assunto pouco explorado no contexto da psiquiatria, pois a maioria das pesquisas preocupa-se em determinar apenas epidemiologicamente as relações existentes entre as variáveis e desfechos, como por exemplo, a relação familiar em parentes de primeiro e segundo graus com a prevalência das alucinações, delírios e assim chamadas de experiências psicóticas. ${ }^{1,6}$ Neste sentido entende-se a necessidade de ampliar 0 conhecimento a respeito das experiências das crianças que lidam de maneira positiva com as vozes. ${ }^{14}$

As alucinações auditivas verbais ou escuta das vozes são comumente associadas à psicose, contudo uma parte da população geral, inclusive crianças vivenciam com frequência e sem sofrimento. Essas experiências não clínicas podem oportunizar o estudo dos mecanismos das alucinações para além de sintomas clínicos. ${ }^{3,7}$ Recentemente algumas teorias ${ }^{1,7}$ apóiam que a escuta das vozes decorre de um desequilíbrio entre as expectativas prévias e informações sensoriais, entretanto permanece desconhecido se este desequilíbrio interfere ou influencia o processo de percepção auditiva das vozes. ${ }^{17}$

A maioria das pesquisas argumenta sobre a necessidade de qualificação da escuta terapêutica no momento da entrevista, afirmam a importância de revisar a realização de testes laboratoriais e psicológicos e também as escalas de avaliação, contudo acredita-se que a escuta das
ISSN $2236-1987$

vozes como fenômeno complexo na infância necessita que os profissionais possam ir além de cuidados prescritivos, justamente porque sua complexidade exige uma grande capacidade da equipe multiprofissional para lidar com todo o contexto da criança e sua(s) voz(es). ${ }^{15}$

É incontestável a necessidade de reformulação das práticas de cuidado na enfermagem em saúde mental, sobretudo na abordagem com crianças que experimentam o fenômeno da escuta das vozes. ${ }^{1,3,11}$

\section{CONSIDERAÇÕES FINAIS}

O presente estudo evidenciou que a experiência da escuta das vozes na infância é percebida como uma sintomatologia relacionada à esquizofrenia, estabelecendo critérios de tratamento a partir de referenciais tradicionais e biomédicos.

A partir da análise dos artigos incluídos nesta revisão integrativa identificou-se uma produção incipiente de pesquisas na área de novas abordagens em saúde mental, relacionadas à escuta das vozes na infância, a maioria dos estudos encontrados manteve 0 foco nos aspectos epidemiológicos relacionando fatores genéticos e ambientais interferindo no processo de saúde/doença.

Pretende-se que a leitura deste artigo contribua com o redirecionamento das práticas da enfermagem, no sentido de oferecer subsídios sobre como lidar com a escuta das vozes na infância.

Faz-se necessária a continuação de pesquisas que possam oferecer, aos 


\section{ISSN 2236 - 1987}

profissionais, novas possibilidades na assistência as crianças ouvidoras de vozes ao subverter a lógica impressa na patologia. Neste sentido, considera-se que esta revisão integrativa contribui para fortalecer o campo psicossocial infanto-juvenil.

\section{REFERÊNCIAS}

1 Cardano M, Lepori G. Udire La vochi degli dei: l'esperienza Dell grupo voci. Milano: Franco Angeli; 2012.

2 Waters F, Fernyhough C. Hallucinations: A systematic review of points of similarity and difference across diagnostic classes. Schizophr bull [Internet]. 2017 Jan[cited 2018 Aug 05];43(1):32-43. Available from: https://www.ncbi.nlm.nih.gov/pmc/a rticles/PMC5216859/

3 Kantorski LP, Antonacci MH, Andrade APM, Cardano M, Minelli M. Grupos de ouvidores de vozes: estratégias e enfrentamentos. Saúde Debate [Internet]. 2017 [acesso em 2018 ago 05];41(115):1143-55. Disponível em: http://www.scielo.br/pdf/sdeb/v41n 115/0103-1104-sdeb-41-115-1143.pdf

4 Intervoice - International Hearing Voices Projects. Children and young people [Internet]. [cited 2018 mar 24]. Available from: http://www.intervoiceonline.org

5 Mendes KDS, Silveira RCCP, Galvão CM. Revisão integrativa: método de pesquisa para a incorporação de evidências na saúde e na enfermagem. Texto \& contexto enferm [Internet]. 2008 out/dez[acesso em 2018 ago 05];7(4):758-64. Disponível em: http://www.scielo.br/pdf/tce/v17n4/ 18.pdf
6 Schroeder K, Langeland W, Fisher H, Huber C, Shäfer I. Dissociation in patients with schizophrenia spectrum disorders: what is the role of different types of childhood adversity? Comprehensive psychiatry [Internet]. 2016 [cited 2018 Aug. 05];(68):201-8. Available from: https://www.sciencedirect.com/scien ce/article/pii/S0010440X15300420?via \%3Dihub

7 Remberk B. Clinical significance of psychotic-like experiences in children and adolescents. Psychiatr pol [Internet]. 2017 [cited 2018 Aug 05];51(2):271-82. Available from: http://psychiatriapolska.pl/uploads/i mages/PP_2_2017/ENGver271Rember k_PsychiatrPol2017v51i2.pdf

8 Alderson-day B, Lima CF, Evans S, Krishnan S, Schanmugalingam $P$, Fernyhough $\mathrm{C}$, et al. Distinct processing of ambiguous speech in people with non-clinical auditory verbal hallucinations. Brain [Internet]. 2017 [cited 2018 Aug 05];140:2475-89. Available from: https: / / academic.oup.com/brain/arti cle/140/9/2475/4085330

9 Lancaster T, Hall J. Altered intraand inter-network dynamics reflect symptom dimensions in childhoodonset schizophrenia. Brain [Internet]. 2016 jan[cited 2018 Aug 05];1(139):102. Available from: https: / /academic.oup.com/brain/arti cle/139/1/10/2468813

10 Haug $E$, Oie $M$, Andreassen OA, Bratlien $U$, Nelson B, Aas $M$, et al. Anomalous self-experience and childhood trauma in first-episode schizophrenia. Comprehensive psychiatry [Internet]. 2015 Jan[cited 
ISSN 2236 - 1987

2018 Aug 05];56:35-41. Available from: https://www.sciencedirect.com/scien ce/article/pii/S0010440X14002958?via \%3Dihub

11 Jeppesen P, Larsen JT, Clemmensen L, Munkholm A, Rimvall MK, Rask CU, et al.The CCC2000 birth cohort study of register-based family history of mental disorders and psychotic experiences in Offspring. Schizophr bull [Internet]. 2015 Sept[cited 2018 Aug 05];41(5):1084-94. Available from: https://academic.oup.com/schizophr eniabulletin/article/41/5/1084/19194 91

12 Ahn K, An SS, Shugarty YY, Rapoport $\mathrm{JL}$. Common polygenic variation and risk for childhood-onset schizophrenia. Mol psychiatry [Internet]. 2016 Jan[cited 2018 Aug 05];21:94-6. Available from: https://www. nature.com/articles/mp 2014158

13 Zhou D, Gochman P, Broadnax D, Rapoport JL, Ahn K. 15q13.3 Duplication in two patients with childhood-onset schizophrenia. Am $j$ med genet $b$ neuropsychiatr genet [Internet]. 2016 Sept[cited 2018 Aug 05];171:777-83. Available from: https://onlinelibrary.wiley.com/doi/f ull/10.1002/ajmg.b.32439

14 Tiffin PA, Kitchen CEW. Incidence and 12-month outcome of childhood non-affective psychoses: british national surveillance study. The british journal of psychiatry [Internet]. 2015 [cited 2018 Aug 05];206:517-8. Available from: https://www.cambridge.org/core/ser vices/aop-cambridgecore/content/view/11905987B7319DF ACAB7714DCBEF51AB/S0007125000279 166a.pdf/incidence_and_12month_out come_of_childhood_nonaffective_psyc hoses_british_national_surveillance_st udy.pdf

15 Ruffell T, Azis M, Hassanali N, Ames $\mathrm{C}$, Browning $\mathrm{S}$, Bracegirdle $\mathrm{K}$, et al. Variation in psychosocial influences according to the dimensions and content of children's unusual experiences: potential routes for the development of targeted interventions. Eur child adolesc psychiatry [Internet]. 2016 Mar[cited 2018 Aug 05];25:311-9. Available from: https://link.springer.com/article/10. 1007/s00787-015-0739-8

16 Sikich L. Diagnosis and evaluation of hallucinations and other psychotic symptoms in children and adolescentes. Child adolesc psychiatric clin n am [Internet]. 2013 Oct[cited 2018 Aug 05];22:655-73. Available from:

https: / / www.sciencedirect.com/scien ce/article/pii/S1056499313000400?via \%3Dihub

17 Agnew-blais JC, Buka SL, Fitzmaurice GM, Smoller JW, Goldstein JM, Seidmann LJ. Early childhood IQ trajectories in indivuduals later developing schizophrenia and affective psychoses in the new england Family studies. Schizophr bull [Internet]. 2015 July[cited 2018 Aug 05];41(4):817-23. Available from: https://www.ncbi.nlm.nih.gov/pmc/a rticles/PMC4466188/

Data de publicação: 19/09/2018 